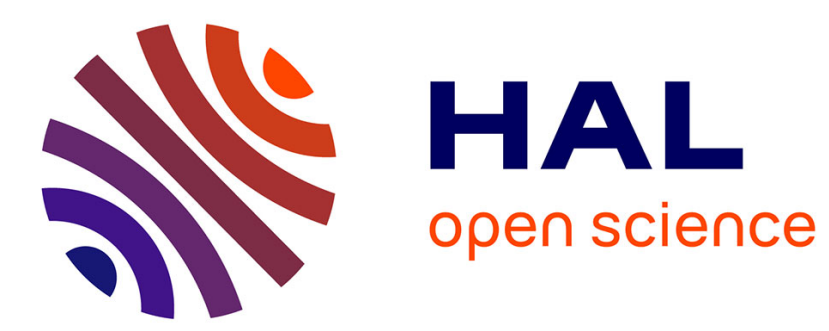

\title{
Spiele und Spielen im römischen Ägypten: Die Zeugnisse der verschiedenen Quellenarten
}

Rudolf Haensch

\section{To cite this version:}

Rudolf Haensch. Spiele und Spielen im römischen Ägypten: Die Zeugnisse der verschiedenen Quellenarten. Archimède: archéologie et histoire ancienne, 2019, 6, pp.175-185. halshs-02927500

\section{HAL Id: halshs-02927500 \\ https://shs.hal.science/halshs-02927500}

Submitted on 1 Sep 2020

HAL is a multi-disciplinary open access archive for the deposit and dissemination of scientific research documents, whether they are published or not. The documents may come from teaching and research institutions in France or abroad, or from public or private research centers.
L'archive ouverte pluridisciplinaire HAL, est destinée au dépôt et à la diffusion de documents scientifiques de niveau recherche, publiés ou non, émanant des établissements d'enseignement et de recherche français ou étrangers, des laboratoires publics ou privés. 


\section{ARCHIMĖDE No6

1 DOSSIER THÉMATIQUE :

HISTOIRES DE FIGURES CONSTRUITES : LES FONDATEURS DE RELIGION

DOSSIER THÉMATIQUE :

JOUER DANS L'ANTIQUITÉ : IDENTITÉ ET MULTICULTURALITÉ

GAMES AND PLAY IN ANTIQUITY: IDENTITY AND MULTICULTURALITY

71 Véronique DASEN et Ulrich SCHÄDLER

Introduction

\section{EGYPTE}

75 Anne DUNN-VATURI

Aux sources du « jeu du chien et du chacal »

89 Alex DE VOoGT

Traces of Appropriation: Roman Board Games in Egypt and Sudan

100 Thierry DEPAULIS

Dés coptes ? Dés indiens?

MONDE GREC

113 Richard. H.J. ASHTON

Astragaloi on Greek Coins of Asia Minor

127 Véronique DASEN

Saltimbanques et circulation de jeux

144 Despina IGNATIADOU

Luxury Board Games for the Northern Greek Elite

160 Ulrich SCHÄDLER

Greeks, Etruscans, and Celts at play

MONDE ROMAIN

\section{Rudolf HAENSCH}

Spiele und Spielen im römischen Ägypten: Die Zeugnisse der verschiedenen Quellenarten

186 Yves MANNIEZ

Jouer dans l'au-delà ? Le mobilier ludique des sépultures de Gaule méridionale et de Corse ( $V^{\text {e }}$ siècle av. J.-C. - Ve siècle apr. J.-C.)

199 Mark Anthony HALL

Whose Game is it Anyway? Board and Dice Games as an Example of Cultural Transfer and Hybridity 


\title{
SPIELE UND SPIELEN IM RÖMISCHEN ÄGYPTEN: DIE ZEUGNISSE DER VERSCHIEDENEN QUELLENARTEN
}

\author{
Rudolf HAENSCH \\ Professor \\ Kommission für Alte Geschichte und Epigraphik des \\ Deutschen Archäologischen Instituts \\ rudolf.haensch@dainst.de
}

ABSTRACT

\section{Games and Play in Roman Egypt: The Evidence of the Different Types of Sources}

Soldiers were - and are always - players, partly in order to pass the time. Is this statement true for all professional soldiers of the Roman army in the Imperial period? This article will gather as exhaustively as possible the literary and archaeological sources concerning the practices of games and play in the Roman army. On this base, we will examine the popularity of different games and question if some games were produced specifically for the soldiers. This paper will thus present for the first time the papyrological (and literary) evidence concerning toys and games in Greek and Roman Egypt, discuss the archaeological evidence for gaming in the forts and quarries in the Eastern desert, taking also into consideration the evidence from other Roman military sites all over the Roman empire, present the references to games and gaming materials invented in Greek and Roman Egypt.
Les jeux et pratiques ludiques en Égypte à l'époque romaine : le témoignage des différents types de sources

Les soldats ont été - et sont toujours - des joueurs, en partie pour faire passer le temps. Est-ce vrai pour tous les soldats professionnels de l'armée romaine du Haut Empire ? Cet article réunit de manière aussi complète que possible les sources littéraires et archéologiques sur les jeux et les pratiques ludiques dans l'armée romaine. Nous examinerons la popularité des différents jeux en nous interrogeant sur la production de certains jeux spécifiquement pour les soldats. Cet article vise les trois objectifs suivants : il entend présenter les témoignages papyrologiques (et littéraires) relatifs aux jouets et aux jeux dans l'Égypte durant les époques grecque et romaine, discuter les témoignages archéologiques en provenance des établissements militaires et des carrières du désert oriental en Égypte et les comparer avec les découvertes du même type provenant d'autres camps romains, présenter les références aux jeux et aux jouets inventés en Égypte au cours de ces époques.
MOTS-CLÉS

Fortins du désert oriental, genius ludi, icosaèdre, Juifs, ostraca, papyri,

lettres privées, armée romaine, schola. 
Papyri gelten zu Recht als der Typ schriftlicher Zeugnisse der Antike, der die alltägliche Realität am unmittelbarsten wiedergibt, und die Region, in der die meisten gefunden wurden, Ägypten, als der Teil des römischen Reiches, über den wir in dieser Hinsicht am meisten wissen. Aus keiner anderen Provinz sind so viele Texte erhalten geblieben, die als Verträge, Briefe und Notizen wie die Masse der heutigen Schriftstücke aus den Notwendigkeiten des Alltags heraus entstanden. Die bewusste Gestaltung, die nicht nur für die Hohe Literatur, sondern auch für Texte von Inschriften oder auf Münzen von zentraler Bedeutung war, spielte bei den Papyri eine verhältnismäßig geringe oder gar keine Rolle. Man erwartet daher auch eine Reihe von Informationen über ein so alltägliches Phänomen wie das Spielen.

Bevor aber erörtert werden kann, inwieweit diese Erwartung tatsächlich erfüllt wird, sind einige Präzisierungen nötig. Es wird im Folgenden nicht um die großen organisierten, öffentlichen Spiele, die Iudi cir-

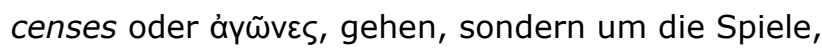
denen sich die Bewohner der Provinz einzeln oder in der Gruppe im Alltag widmeten. Die großen Spiele sind bekannt, gut untersucht und nicht für die Provinz spezifisch. In den Blick genommen werden alle "Bewohner" der Provinz mit ihrem jeweils ganz unterschiedlichen kulturellen Hintergrund, gleich ob sie 1. Bewohner des flachen Landes, der Chora, waren, also z. T. ägyptischer oder (ursprünglich) griechischer Herkunft, und unter römischer Herrschaft unterschiedliche Rechte hatten in Abhängigkeit davon, ob sie aus einer der etwa 48 Gauzentren stammten oder außerhalb dieser geboren waren; 2. die Bewohner der sich selbständig verwaltenden griechischen Städte wie Naukratis, Ptolemais Hermou und - mit Einschränkungen - Antinoupolis sowie des Sonderfalls Alexandria (einschließlich des großen jüdischen Bevölkerungsanteils insbesondere in dieser Stadt bis zum Jüdischen Aufstand unter Traian) und 3. die im Land am Nil lebenden cives Romani [1]. Erst mit der Einführung von Stadträten in den Gauzentren unter Septimius Severus und der Verleihung des römischen Bürgerrechtes an fast alle freien Bewohner des Imperium Romanum unter seinem Sohn egalisierten sich die angeführten Unterschiede.

Einerseits waren alle Ethnien schon vor 212 n. Chr. vielfältig miteinander verwoben. Andererseits wurden im Laufe der ersten zwei Jahrhunderte der Kaiserzeit immer mehr cives Romani nicht als solche geboren und stammten auch nicht aus Rom oder Italien. Solche cives Romani darf man daher im Gegensatz zu den Bewohnern Italiens und Roms nicht als typische „Römer" betrachten, sondern als mit dem römischen Bürgerrecht ausgestattete indigene Provinzialen, die in ganz unterschiedlicher Weise je nach regionaler Situation und individuellem Lebensschicksal römische Sitten und Gebräuche übernommen hatten.

Schließlich ist noch zu unterstreichen, dass es sich bei den folgenden Ausführungen um einen ersten Survey handelt [2]. Es wurde zusammengestellt, was dem Autor aus den unterschiedlichsten Quellen, papyrologischen, literarischen und in situ erhaltenen archäologischen Funden, über Spiele und Spielen im römischen Ägypten [3] bekannt wurde. Angesichts der völlig verstreuten Informationen und der Tatsache, dass von dem archäologischen Material wohl noch vieles unpubliziert in Museen und Sammlungen schlummert, kann diese erste Zusammenstellung nicht vollständig sein.

\section{DAS ZEUGNIS DER PAPYRI}

Gleich ob man die griechischen Begriffe für Spielzeug im Generellen, für bestimmte Spiele oder für Spielgegenstände wie Würfel etc. in die papyrologischen Datenbanken (Heidelberger Gesamtverzeichnis der Papyrusurkunden, Trismegistos) eingibt oder in den Wörterbüchern nachschlägt, die Ernte fällt unerwartet mager aus. Man findet kaum etwas, ja oft sogar nur Belege für die Verwendung eines bestimmten Wortes in anderen Bedeutungen. Im Falle des grie-

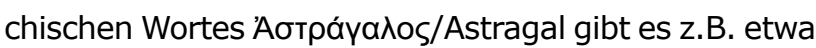

[1] Den besten Einstieg in die administrative Struktur des römischen Ägyptens bietet JöRDENS 2009.

[2] Einen Schwerpunkt auf die Funde aus dem ägyptisch-nubischen Grenzgebiet und speziell dem Gebel el-Silsila legen die Autoren von CRIST \& DUNN-VATURI \& DE VOoGT 2016 in ihrem Kapitel "Roman Board Games Crossing the Borders of Egypt" (p. 125-150).

[3] Die Studie beschränkt sich aber auf das griechisch-römische Ägypten und behandelt nicht das gesamte Römische Reich und nimmt auch nicht das Land am Nil in allen seinen Perioden in den Blick. Wollte man dies versuchen, so würde wohl eine kommentierte Bibliographie entstehen, wie sie HüBNER 1992 für Iudaea / Palaestina vorlegte. 


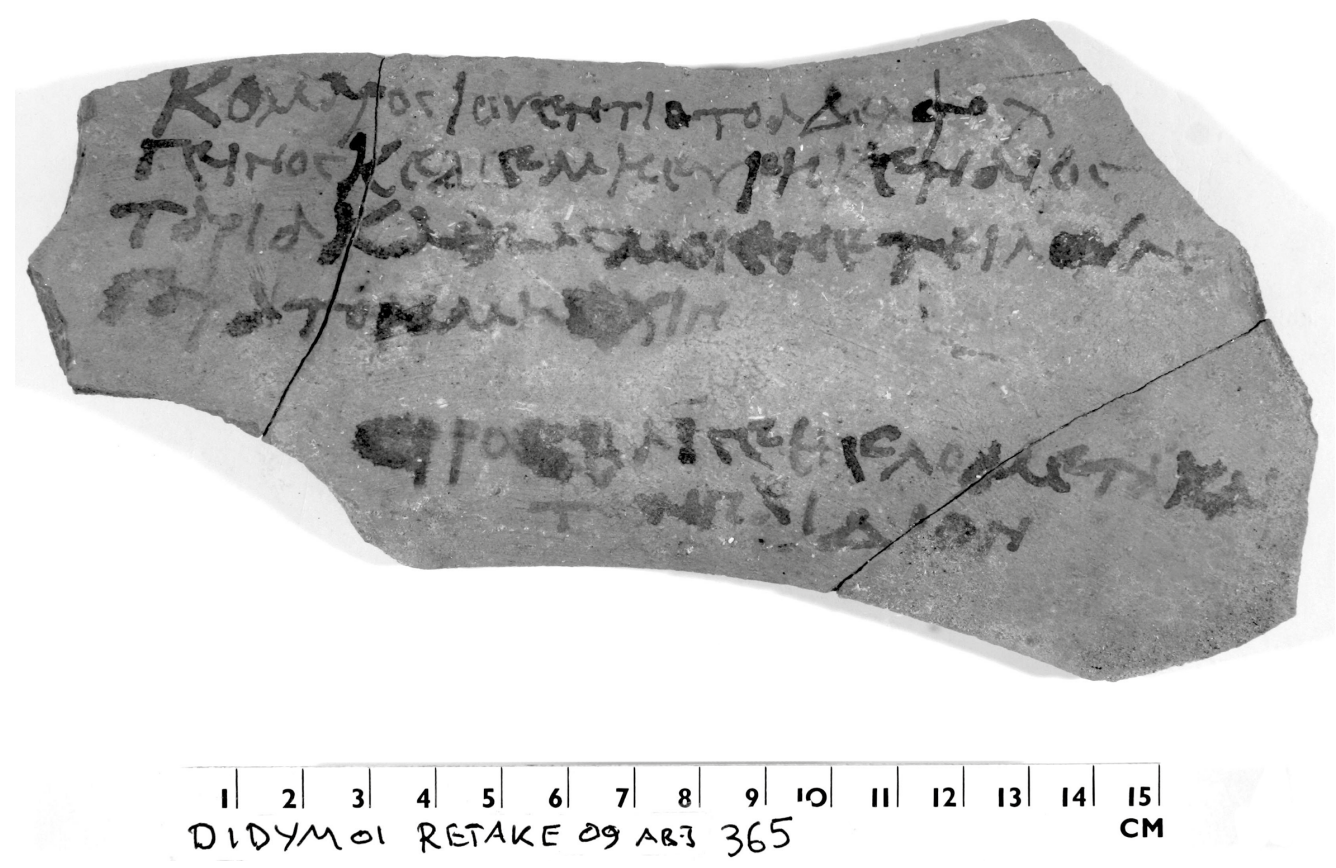

30 Belege aus dem griechisch-römischen Ägypten. Aber sie finden sich alle in den sogenannten Signalements [4] von Rechtsurkunden, also dort wo auffällige körperliche Merkmale von Vertragsparteien genannt wurden, um diese sicher identifizieren zu können. Mit anderen Worten: Die betreffenden Personen in diesen Vertragsurkunden hatten alle einen auffälligen Knöchel. Übrig bleibt nach einer solchen Suche nur wenig und zwar bezeichnenderweise in den beiden Haupttypen der Dokumente von Privatpersonen: Ihren Briefen und ihren Abrechnungen und Aufstellungen. Vier Briefe aus dem griechisch-römischen Ägypten sprechen vom Spielen und Spielmaterial. Drei davon machen deutlich, welche Mühen man schon auf sich nehmen musste, um ein neues Set von Würfeln zu bekommen. Schon auf den ersten Blick fällt die ganz unterschiedliche Zeitstellung der Briefe auf und ebenso dass sie nicht von den verhältnismäßig vielen gut-situierten (Groß)bauern, Handwerkern und Kaufleuten mit zumindest gewissen Schreib- und Lesefähigkeiten geschrieben wurden, sondern von Angehörigen herausragender Schichten. Bei den ersten drei Briefen handelt es sich um Schreiben aus der frühen Ptolemäerzeit, genau gesagt aus der Mitte des 3. Jh. v. Chr., und damit eigentlich außerhalb des hier zugrunde gelegten Zeitraumes. Sie stammen aus einem der umfangreichsten erhaltenen Dokumentenkomplexe des antiken Ägyptens, dem sogenannten Archiv des Zenon. Dieser Zenon war einer der Gutsverwalter eines der engsten Mitarbeiter des 2. Ptolemäerkönigs, Apollonios aus Kaunos, der wiederum lange Jahre die Finanzverwaltung in Ägypten leitete [5]. In einem Schreiben an Apollonios wird die eigene Unsicherheit mit der Unruhe verglichen, die Würfelspieler zeigen würden, wenn sie ahnten, dass sie in ein Spiel mit gezinkten Würfeln geraten wären [6]. Konkreter sind zwei andere Schreiben. Einmal wurde Zenon von einem nicht mehr zu identifizierenden Korrespondenzpartner berichtet, man hätte sich bei einem Handwerker, der auch für den Königshof

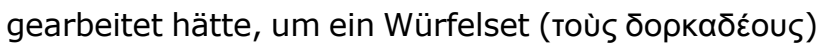
aus Gazellenknochen bemüht. Dieses würde ein oder höchstens zwei Bronzemünzen kosten [7]. In einem anderen Schreiben an Zenon berichtet ein gewisser Epharmostos, vielleicht sein jüngerer Bruder, ein Mann namens Eutychos hätte ihm nicht das Würfelspiel (Toùs боркабє́ouৎ) ausgehändigt, das Zenon inm über Eutychos habe zukommen lassen wollen. Er selbst aber hätte sich bei einem dritten ein solches besorgt und dieses einer vierten Person anvertraut [8]. Würfel waren offensichtlich sehr begehrt!

Genau dies geht auch aus einem etwa 400 Jahre späteren, diesmal auf einem Ostrakon, einer Tonscherbe, festgehaltenen Schreiben hervor (Fig. 1). Es war an einen Soldaten namens Iuventius gerichtet, der in einem kleinen Fort, einem praesidium namens

[4] HüBSCH 1967.

[5] Zu Zenon Z. B. Orrieux 1983a und OrrieuX $1983 b$.

[6] PSI IV 418; im Netz abrufbar unter TM (= trismegistos) Nr. 2101

[7] PSI IV 444 (auch greifbar als P. Cair. Zenon I 59019); im Netz: TM Nr. 679. Dazu KIDD 2017, 125.

[8] PSI IV 331; im Netz: TM Nr. 2025. 
Didymoi, in der östlichen Wüste an einer der Straßen zum Roten Meer stationiert war [9]. Dieser Soldat hatte anscheinend einen mutmaßlichen Kameraden namens Komaros gebeten, ihm ein Würfelset (óorápı́a) bei einer dritten Person zu besorgen. Doch dieser ließ mitteilen, er selbst hätte keines. Dem Namen nach müsste man in Iuventius einen römischen Bürger sehen. Doch sind in Ägypten in der Frühen und Hohen Kaiserzeit (hier konkret zwischen 110 und $115 \mathrm{n}$. Chr.) die Namen römischer Soldaten kein Indiz für deren ethnische Herkunft. Offensichtlich wollte man in römischen Armeematrikeln keine aus römischer Sicht unaussprechlichen ägyptischen Namen verzeichnen und benannte Rekruten serienweise bei der Aufnahme in die Armee um [10].

Vier Briefe sind nicht viel. Aber sie zeigen bezeichnenderweise Interesse am Würfelspiel in zwei Milieus, in denen es in der gesamten abendländischen Geschichte beliebt war: den Soldaten [11] und den hohen Administrationsträgern, also sozialen Gruppen, die über die Mittel und die Zeit für dieses und andere Spiele verfügten, und einen erheblichen Teil ihres Lebens bei gemeinsamen Gelagen verbrachten.

In die gleichen Milieus führen auch die beiden Aufstellungslisten, in denen Spielgerät erscheint: P. Oxy. IV 736 [12] überliefert eine nach Tagesdaten geordnete Ausgabenliste. Der Schrift nach dürfte sie um die Zeitenwende entstanden sein. Wie fast immer bei solchen Dokumenten wissen wir nicht präzis, wer diese Aufstellung machte - weil man dies, genau wie heute, auf solchen kurzlebigen Zusammenstellungen nicht verzeichnete. Am ehesten möchte man an ein militärisches Milieu denken, denn es findet sich nicht nur der Begriff

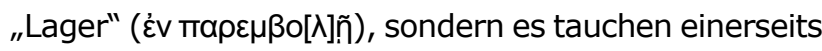
viele lateinische Namen (Gemellus, Prima, Secunda, Caecilius) auf. Andererseits spricht man aber auch von der Mumie der Tochter der Phna (also einer Trägerin eines ägyptischen Namens). Eine solche kulturelle Vermischung zwischen Trägern römischer und ägyptischer Namen ist zu diesem frühen Zeitpunkt am ehesten im Zusammenhang mit in Ägypten stationierten Truppen zu erwarten. In dieser Liste ist unter einem bestimmten Tagesdatum neben Ausgaben für Bier (drei Obolen), Würzsauce und Granatäpfel (je eine Obole) auch eine halbe Obole als Ausgabe für Spielzeug und anderes "der

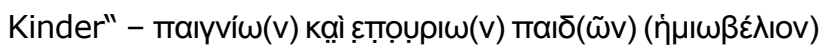
- verzeichnet. Um was für eine Art von Spielzeug es sich genau handelt, bleibt offen.

Das gilt ebenso für ein zweites, wesentlich späteres, nämlich aus dem späten 7. oder frühen 8. Jh. n. Chr. stammendes, Register aus dem Bereich eines gröBeren privaten Wirtschaftsbetriebes [13]. In diesem Fall gab man einer bestimmten, nicht näher einordbaren Person einen follis, also die zentrale spätantike

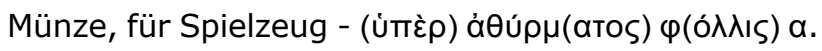
Schließlich wird in einem Privatbrief des 3. Jh. n. Chr. im Zusammenhang mit der Erwähnung eines kleinen Jungen die Übersendung von acht Stück Spielsachen angekündigt [14].

Die Zahl der Papyri und Ostraka, in denen Spielen und Spielgerät erwähnt werden, ist also gering und die ihnen zu entnehmenden Informationen spärlich. Unter den bisher publizierten Texten fehlt insbesondere einer, der ein Pendant zu einem im Schutthügel des Legionslagers Vindonissa (in der heutigen Schweiz) gefundenen Wachstäfelchen darstellen würde [15]. Mit diesem lud ein Soldat einen Kameraden zum Gastmahl ein. Er kündigte an, es werde reichlich Wein und verschiedene Spiele geben. Er fuhr fort: „Morgen, bei den wirkmächtigsten genii des Spiels, würde er den Würfelbecher wie ein Schwert schwingen (cras per genios potissimos ludi crispo orcam sicut gladium)". Einladungen sind auch in Ägypten ein keineswegs seltener Dokumententyp. Insofern ist keineswegs ausgeschlossen, dass auch aus dieser Provinz einmal ein lateinisch- oder griechischsprachiges Pendant für ein entsprechendes Einladungsschreiben eines dort stationierten Soldaten bekannt wird. Die bisher nur aus dem Windischer Schreibtäfelchen bekannten genii des Spiels mögen auch an anderen Orten beschworen worden sein. Nur selten dürfte der Gewinn im Spiel so groß gewesen sein, dass man auch nur erwog, dieser
[9] O.Didymoi 437; im Netz: TM Nr. 144998.

[10] Z. B. HAENSCH 2012, p. 73.

[11] Vgl. VoGT 2012, p. 100-103. Der spätrepublikanische Autor Varro ging daher nach einer griechischen Sage davon aus, dass die Brettspiele im Trojanischen Krieg erfunden worden seien, um Meutereien aus Langeweile zu verhindern (ad comprimendos otiosi seditiones exercitus; Servii grammatici in Vergilii Aeneidos commentarius, II 81); zur griechischen Überlieferung: KARASU 1973 und jetzt KIDD 2017, 128. Vgl. auch die Darstellung bei Iuvenalis Saturae, VIII 9-12.

[12] P. Oxy. IV 736; im Netz greifbar unter TM Nr. 20436.
[13] SB XVI 12254; im Netz greifbar als TM Nr. 38652.

[14] PSI IX 1080 = BAGNALL \& CRIBIORE 2006,

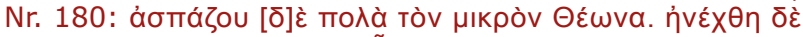

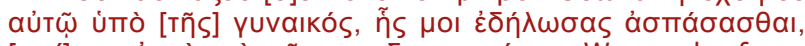

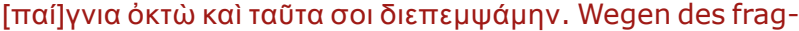
mentarischen Zustandes von P. Ryl. II 237 muss fraglich bleiben, ob пغ่үvıov (I. naiyvıov) in Zeile 13 tatsächlich einen Gegenstand bezeichnet oder im übertragenen Sinn gemeint war. Schließlich fragt sich, ob O. Claud. 1 (auch TM Nr. 29810), das von den Herausgebern als Kuriosum betrachtet wird, nicht in den Zusammenhang eines Ratespieles wie Artiasmos oder Posinda gehört.

[15] Speidel 1996, Nr. 45. 


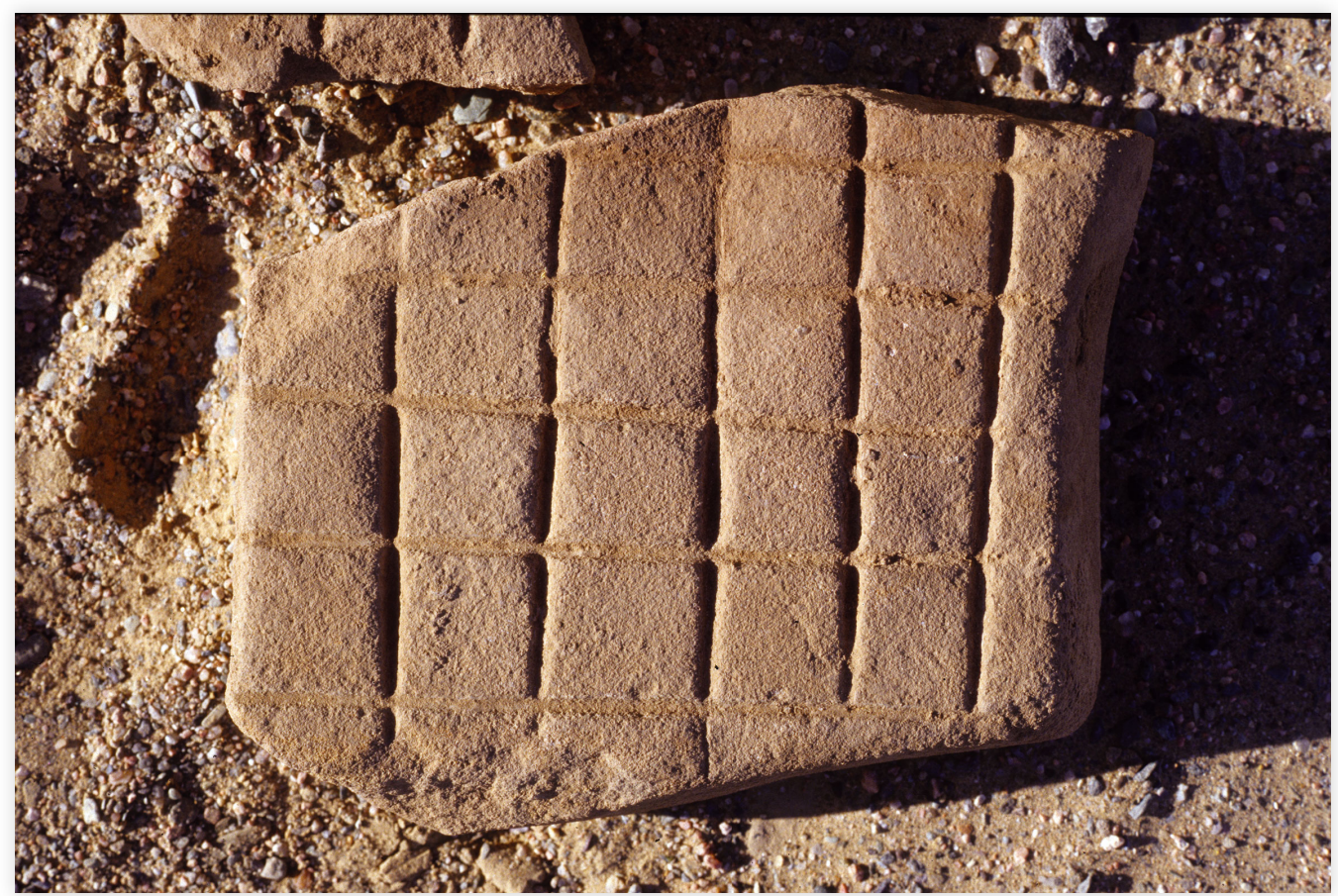

Figure 2

Ludus Latrunculorum aus Krokodilo (veröffentlicht bei Matelly 2003 p. 594 mit fig. 278; Foto: Adam Bülow-Jacobsen; mit freundlicher Erlaubnis von Hélène Cuvigny). göttlichen Wirkkraft einen kleinen oder größeren steinernen Altar zu stiften und damit sich so bedanken, wie man dies im Imperium Romanum gerne tat und wie dies von allen antiken Formen solcher Dankbarkeit heute noch am besten zu fassen ist.

Insgesamt dürfte es beim näheren Überlegen kein Zufall sein, dass man in den Papyri so wenig Spuren von Spielen und vom Spielen findet, auch wenn in mancher unpublizierten Abrechnung oder in manchem bisher nicht veröffentlichtem Privatbrief noch Hinweise zu erwarten sind. Papyri wurden zwar aus den Notwendigkeiten des Alltags heraus beschrieben. Aber sie waren doch an gewichtigere Anlässe im Alltagsleben gebunden. Keineswegs wurde in ihnen wie in den Briefen des letzten Jahrhunderts, in e-mails oder in sogenannten sozialen Netzwerken wie Facebook über alles und jedes berichtet. Es gibt z.B. auch fast keine Liebesbriefe aus dem griechisch-römischen Ägypten. Die Zahl der Briefe, die selbst ein literater oder halbliterater Soldat oder Angehöriger der schwer zu fassenden „Mittelschichten" im Laufe seines Lebens schrieb, war sehr begrenzt. Das zeigen die Fälle, in denen anscheinend das ganze derartige Material einer Person, in der Sprache der Papyrologen deren Archiv [16], erhalten blieb. Der Inhalt der Briefe war auf bestimmte

\section{[16] JÖRDENS 2001.}

[17] Zu den Funden aus dem jenseits der Grenze liegenden Gebel el-Silsila oben Anm. 2.

[18] Vgl. als Überblick BagnaLL \& RATHBOne 2004, p. 78-292; GATES-FOSTER 2012 mit der dort genannten Literatur und die hier in den folgenden Anmerkungen genannten exzellenten Einzelpublikationen.
Themen fokussiert - vor allem wurden Verwandte und Bekannte gegrüßt und am Ort nicht zu bekommende Güter erbeten.

Zusammenstellungen aller Art aber, in denen, wie die hier vorgeführten Zeugnisse zeigen, noch am ehesten Hinweise auf Spielutensilien zu erwarten sind, gehören zu dem bisher am wenigsten beachteten und publizierten Typ von Dokumenten. Dazu bieten sie sowohl unter historischen wie auch unter philologischen Gesichtspunkten für Papyrologen vermeintlich zu wenig neue Erkenntnisse.

\section{ARCHÄOLOGISCHE FUNDE IN SITU}

Damit sind für das römische Ägypten am Ort erhaltene archäologische Funde die wichtigste Quelle für antike Spiele. Wie in anderen Provinzen gilt aber auch für Ägypten, dass gerade Spielgerät zu den Objekten gehört, deren genaue Provenienz und Zeitstellung nicht oder nur mit großer Unsicherheit zu bestimmen sind, wenn sie, wie es üblicherweise der Fall ist, nicht bei Grabungen oder wissenschaftlichen Surveys entdeckt werden, sondern nur über den Antiquitätenhandel bekannt werden [17].

Ganz anders war dies freilich bis vor wenigen Jahren in einem bestimmten Teile Ägyptens, nämlich der wegen ihrer unwirtlichen Bedingungen in Mittelalter und Neuzeit kaum besiedelten östlichen Wüste. Unter römischer Herrschaft war sie aber wegen der vorhandenen Rohstoffe und des Indienhandels mit einem Netz von Stützpunkten für die Ausbeutung der Mineralien und den Schutz der Karawanen versehen worden [18]. 


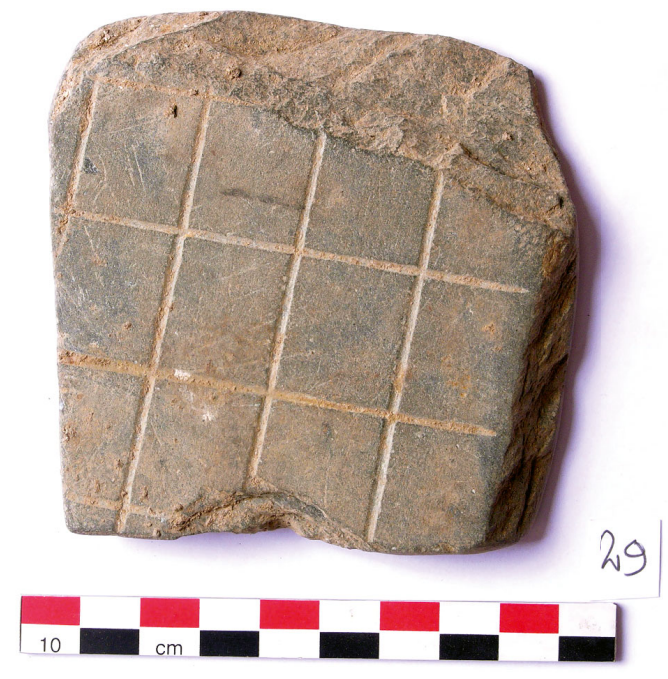

A Figure 3: Ludus Latrunculorum aus Xeron (Foto: Adam Bülow-Jacobsen; mit freundlicher Erlaubnis von Hélène Cuvigny).

1 Figure 4: Würfel und Spielbecher aus Xeron (Foto: Adam Bülow-Jacobsen; mit freundlicher Erlaubnis von Hélène Cuvigny).

$>$ Figure 5: Würfel und Spielbecher aus Umm Balad (Foto: Adam Bülow-Jacobsen; mit freundlicher Erlaubnis von Hélène Cuvigny).

Insbesondere bei den langjährigen Surveys eines französischen Teams um Jean-Pierre Brun, Hélène Cuvigny und Adam Bülow-Jacobsen in der östlichen Wüste ist immer wieder Spielgerät im Rahmen von wissenschaftlichen Projekten vor Ort gefunden und publiziert worden. Das gilt zunächst für die Straße von Koptos am Nil zu dem Hafen Myos Hormos am Roten Meer: Aus Krokodilo, einer 48x48 m grossen Schutzstation (praesidium), die $65 \mathrm{~km}$ von Koptos und 116 $\mathrm{km}$ von Myos Hormos entfernt lag, wurden ein Spielbrett aus Kalkstein mit sehr sorgfältig gearbeiteten Quadraten, wohl für den Ludus latrunculorum (fig. 2), sowie drei latrunculi und ein Würfel bekannt [19]. In Dawwi, einem fast identisch großen, nur kurzfristig im 2. Jh. bestehenden Lager $25 \mathrm{~km}$ vor Myos Hormos, war es eine Kalksteinplatte für ein Dreilinienspiel, am ehesten wohl der Ludus duodecim scriptorum [20].

[19] Matelly 2003, p. 594 mit fig. 278 f.

[20] BRUN 2003, p. 135, p. 184 fig. 174.

[21] CuvignY 2011, fig. 218, 236, 240.

[22] Beispiele für angeblich gezinkte Würfel sind ansonsten aus Heddernheim (LAMER 1927, col. 2023) und Housesteads (FIELDS 2003, p. 55) bekannt. Doch vgl. zur Interpretation SCHMID 1978.

[23] Freundliche Mitteilung von $\mathrm{H}$. Cuvigny.

[24] Freundliche Mitteilung von $\mathrm{H}$. Cuvigny. Zu Umm Balad z. B. BAGNALL \& RATHBONe 2004, p. 288.
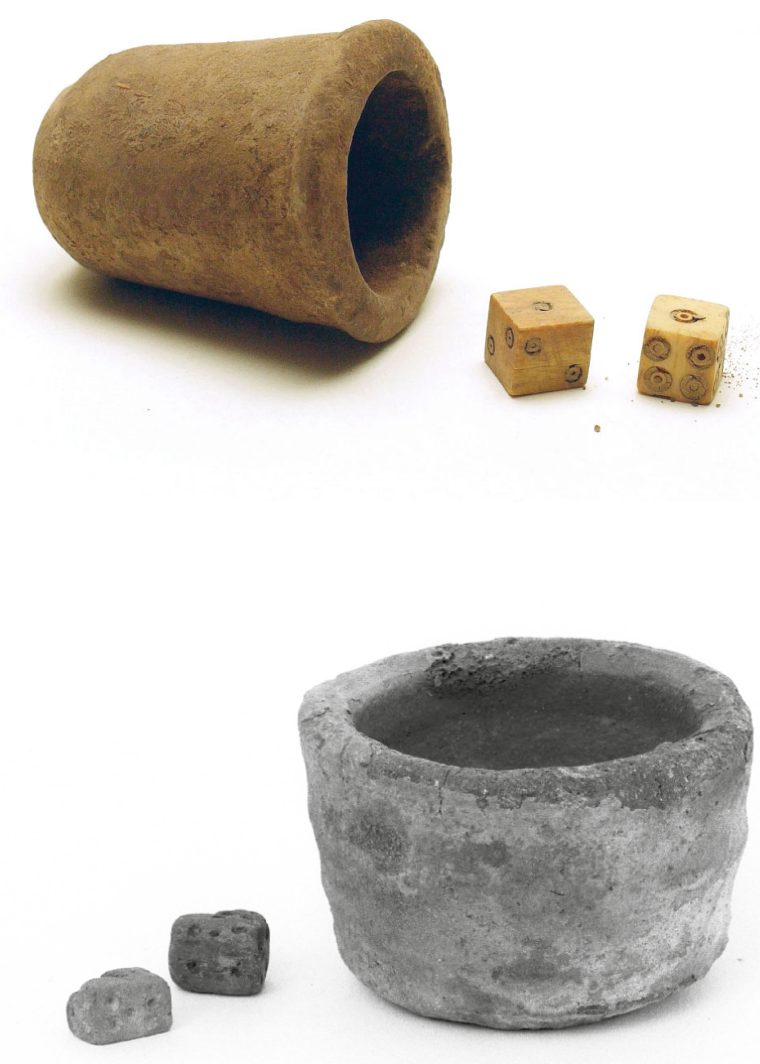

In gleicher Weise wurde auch die zweite große Verbindungsstrecke vom Nil zum Roten Meer, die von Koptos nach Berenike, untersucht: Im dortigen Kleinkastell Didymoi, 54×44 m groß, aus dem der oben erörterte Brief des Iuventius stammte, fand sich in drei Siedlungsschichten Spielgerät. Aus der Zeit um $96 \mathrm{n}$. Chr. stammt ein wiederum recht sorgfältig gearbeiteter, in gleichmäßige Quadrate aufgeteilter steinerner Spieltisch, wohl für den Ludus latrunculorum; aus der Periode zwischen 123 und $150 \mathrm{n}$. Chr. zwei Teile eines entsprechenden in Quadrate aufgeteilten Tisches sowie fünf Teile eines Spiels mit Linien und darüber gesetzten Kreisen, wohl am ehesten der Ludus duodecim scriptorum. Aus der anschließenden Epoche gibt es noch einmal einen Rest eines erneut sorgfältig gearbeiteten Spieltisches mit hintereinander aufgereihten Kreisen, vermutlich auch von einem Ludus duodecim scriptorum [21]. In Xeron, einem praesidium an derselben Straße, fand man neben einem Spielbrett für den Ludus latrunculorum (fig. 3), zwei Würfel, davon einen möglicherweise gezinkten [22], und einen mutmaßlichen Würfelbecher (fig. 4) [23]. Ganz ähnliches Spielmaterial fand sich bei dem recht bald nach seiner Eröffnung aufgegebenen Steinbruch von Umm Balad beim Mons Porphyrites (fig. 5) [24].

Der umfangreichste Fund aber stammt aus einer zwischen 309 und $311 \mathrm{n}$. Chr. errichteten, spätantiken Befestigung für etwa 200 Mann am Roten Meer, 
aus Abu Sha'ar. Dort fanden sich in verschiedenen Fundsituationen insgesamt 19, vielleicht 20 steinerne Spieltische [25]. Sie waren z.T. ad hoc an einem häufig frequentierten Platz an einer geeigneten Stelle eingraviert, dienten teilweise als tragbare Spielbretter und waren in einzelnen Fällen offensichtlich bewusst bei der Gestaltung eines Raumes eingebaut worden. Alle Spieltische sind qualitätsmäßig wesentlich schlechter gearbeitet als die in den kaiserzeitlichen praesidia. Gespielt wurden drei verschiedene Typen von Spielen, der Ludus duodecim scriptorum, der Ludus latrunculorum und vielleicht Mancala [26]. Ob es wirklich einen Spieltisch für Mühle gab, bleibt fraglich. Besonderes Aufsehen hat die These der Ausgräber erregt, man habe ein Spielcasino gefunden [27]. Es handelt sich um einen kleineren rechteckigen Raum, in den an drei Seiten Klinen aus Steinblöcken eingebaut waren. Mitten zwischen den Liegemöglichkeiten gab es einen steinernen Spieltisch, und auf einer anderen Kline war ein Mancala(?)-Spiel eingraviert. Statt aus diesem Befund aber einen bewusst geplanten "gaming room" zu machen, sollte man die zweite von den Ausgräbern angedachte Interpretationsmöglichkeit "a soldier's mess with gaming tables" weiterdenken. Für solche Räume, in denen Soldaten feierten und ihr otium verbrachten, gab es im römischen Heer durchaus einen festen Begriff: Der vermutete "gaming room" ist eine schola, ein Versammlungsort von Soldaten eines bestimmten Dienstranges für ihre dienstfreie Zeit und speziell ihre Feiern [28]. Dass es sich nicht um einen einzigartigen Fund handelt, zeigt eine Ausgrabung vom anderen Ende des Imperium Romanum, im Lager Petavonium der in der Hispania Citerior stationierten ala II Flavia [29].

Insgesamt wird durch diese Funde erneut deutlich, wie verbreitet das Spielen mit Würfeln und Brettspielen in der römischen Armee war [30]. Wie bei Soldaten anderer Epochen war beides offensichtlich ein bevorzugter Zeitvertreib. Ob allerdings in der römischen Armee Spielbretter "als Auftragsarbeit" hergestellt wurden [31], bleibt fraglich. Man hat dies aufgrund eines vergleichsweise handlichen und mit einem Stempel der in Germania inferior stationierten legio XXX Ulpia Victrix versehenen, nach $193 \mathrm{n}$. Chr. gestrichenen, Ziegels vermutet, in den man vor dem Brennen ein Mühlespiel einritzte und der heute in Brühl aufbewahrt wird.

Die römische Armee versorgte ihre Angehörigen mit vielen militärischen und alimentaren Grundgütern. Das zeigt gerade auch ein Spielbrett aus dem Prätorianerlager in Rom, auf dem diese Lebensmittel angeführt werden [32]. Aber dass sich römische Offiziere mit der Frage beschäftigten, wie ihre Soldaten ihre Freizeit spielerisch gestalten sollten, ist doch wohl zu modern gedacht. Die Hypothese wird auch nicht gebraucht, um den Befund zu erklären. Vielmehr ist grundsätzlich davon auszugehen, dass die einzelnen Soldaten Spielbretter aus all dem anfertigten, was es vor Ort am leichtesten gab: Holz, Kalkstein oder - eben beim Dienst in einer Ziegelei - aus Ziegeln und dabei durchaus auch für andere Zwecke bestimmte Materialien "abzweigten".

Es ist zweifellos richtig, dass in einer römischen Provinz nicht nur Soldaten spielten. Vielmehr führt zweierlei zu einer vergleichsweise guten Kenntnislage vom Spielen dieser sozialen Gruppe: Erstens gehören Militärlager zu den relativ am häufigsten und intensivst untersuchten Einrichtungen in einer römischen Provinz. Zweitens lässt sich in solchen Lagern gefundenes Spielgerät viel sicherer einer bestimmten Gruppe zuweisen als Einzelfunde in zivilen Siedlungen (abgesehen von herausragenden Grabbefunden). Damit dürften solche Funde aus Militärlagern in unseren Fundberichten im Vergleich zur antiken Realität überrepräsentiert sein. Das heißt wiederum nicht, dass es nicht gute Gründe gibt, dass Soldaten wie Angehörige von Führungsschichten vergleichsweise oft gespielt haben.

Abgesehen von diesem Teil Ägyptens aber haben systematische Ausgrabungen oder Surveys bisher noch keine große Rolle für die Erforschung des
[25] Mulvin \& Sidebotham 2004.

[26] Dagegen jetzt CRIST \& DUNN-VATURI \& DE VoOgT 2016, p. 128 , p. 131 , die an das 5-Linien-Spiel denken.

[27] Vgl. dazu jetzt Crist \& DunN-VATURI \& DE VoogT 2016, p. 141-142.

[28] Vgl. z. B. MARCATTILI 2005, 319; SCHMidT HEIDENREICH 2013, passim, insbesondere p. 96-100.

[29] Carretero Vaquero 1998.

[30] Ein erster Überblick über die in den Lagern Britanniens gefundenen Spielbretter und Spielsteine bei FIELDS 2003, p. 53-56, vgl. CARRETERO VAQUERO 1998, p. 122; für das römische Deutschland bei LAMER 1927, col. 2018 f.; speziell für Mainz DECKER 1972; für Trier STEINER 1939, der u. a. p. 43 einen Spielstein mit der Aufschrift speculator, also der Bezeichnung für einen bestimmten Typ chargierter Soldaten, anführt. Eine archäologische Analyse der Fundorte von Spielsteinen und Würfeln im Legionslager von Vindonissa bei HoLLIGER \& HOLLIGER 1983, p. 4-16. Vgl. auch einen Befund aus dem Lager der legio X Fretensis in Aelia Capitolina, dem heutigen Jerusalem: HüBNER 1992, p. 65.

[31] So die These von HANEL 1997.

[32] Eine Abbildung bei FITTÀ 1998, p. 172; die Inschrift (Bull. Com. 1877, 89 = Ferrua 2001, Nr. 112): $(H)$ abemus in cena $(m)$ pullum piscem pernam paonem benatores. 
griechisch-römischen Ägyptens gespielt. Dementsprechend selten sind genau zuweisbare Funde von Spielgerät. Ein Team der Universität Michigan untersuchte zwischen 1924 und 1935 Häuser in dem Dorf Karanis im Fayum, primär auf der Suche nach Papyri [33]. Dabei wurden aber auch die übrigen Fundgegenstände für die Zeit vergleichsweise sorgfältig dokumentiert. Daher haben wir z. B. Kenntnis von einem Spielzeugpferd auf einem rollenden Sockel aus einem Haus (B17), das zwischen 135 und 171 n. Chr. von einer vor Ort prominenten Familie bewohnt wurde, deren zentrale Person, ein gewisser Sokrates, u. a. als Steuereinnehmer fungierte. Ein anderes Spielzeugpferdchen wurde in El Hibeh/ Ankyronpolis gefunden [34]. Auch bei einer Reihe von Puppen sind die Fundumstände (häufig Gräber) bekannt allerdings nicht für eine vergleichbar gut erhaltene Stoffpuppe, die Goldschmuck trägt und nach der Haarfrisur in hadrianisch-frühantoninische Zeit gehört, also vergleichsweise präzis zu datieren ist [35].

\section{IM GRIECHISCH-RÖMISCHEN ÄGYPTEN ENTWICKELTES UND ZUERST PRODUZIERTES SPIELGERÄT UND SPIELE}

Im Falle manches Spielgerätes lässt sich mit großer Wahrscheinlichkeit feststellen, dass es im griechisch-römischen Ägypten entstanden, zuerst produziert worden ist und von dort aus seinen Weg durch das Imperium Romanum nahm. So wurde offensichtlich ein von Paul Pendrizet veröffentlichter, knapp $71 / 2 \mathrm{~cm}$ großer, zwanzigeckiger Würfel aus Bronze im Alexandria der ptolemäischen Zeit konzipiert [36]. Seine Seiten waren nämlich jeweils mit einer Zahl und einem Begriff versehen. Die Zahl wurde entsprechend der griechischen Praxis durch einen Buchstaben des Alphabets dargestellt. Die diesen Zahlen zugewiesenen Begriffe sind wegen ihrer unterschiedlichen Herkunft und ihres z. T. engen Bezugs auf das ptolemäische Ägypten und speziell Alexandria aufschlussreiche und sichere Indizien für den Entstehungsort. Einerseits findet man in der gesamten griechischen Welt verbreitete
Wurfbezeichnungen: Der 8 wird der Octopus zugewiesen; der 12 die Stunden; der 16 die Jungfrau (vú $\mu \varphi \eta$ ) und die 17 dem schönen Jüngling (ó kaגós); 23 ist der Wurf des Aphrodite und 25 der des Epheben. Andererseits sind gerade die hohen Würfe nach ptolemäischen Institutionen und Personen benannt: Zur 24 gehört die Königin Berenike, zur 29 der Athlophoros, ein nur in Alexandria gegebenes Priesteramt, zur 35 die Gymnasiarchie, ein, wie wir aus anderen Quellen wissen, in Alexandria besonders prestigeträchtiges und für den Amtsinhaber teures städtisches Amt, und der höchste Wurf gilt dem Soter, wohl dem ersten Ptolemäerkönig. D. h. nicht nur das Material, sondern auch die Beischriften deuten auf einen in der alexandrinischen Sozialstruktur oder gar am Hof hoch angesiedelten Besitzer (und wohl auch Auftraggeber) hin. Typisch alexandrinisch ist aber auch, dass man den niedrigen Wurf 6 mit dem Begriff Пробáßßат(ov) / prosabbaton also Tag vor dem Sabbat - negativ zu charakterisieren suchte; denn Alexandria war von heftigen Spannungen zwischen der griechischen Bevölkerungsmehrheit und den zahlreichen dort ansässigen Juden geprägt.

Der zwanzigeckige Würfel als solcher ist aber auch ein gutes Beispiel für das Wandern solchen Spielgerätes zwischen verschiedenen Kulturen und bei verschiedenen Einsatzformen. Im Louvre gibt es einen Ikosaeder mit lateinischen Zahlen und Buchstaben in alphabetischer Reihenfolge, der wie der alexandrinische wohl beim Würfelspiel eingesetzt wurde - wo wissen wir wieder nicht. In Qaret el-Muzzawaqa in der Oase Dakhleh aber wurde ein Ikosaeder gefunden, dessen zwanzig Seiten in Demotisch mit den Namen von Göttern beschrieben war. Er dürfte wohl bei Zukunftsbefragungen in einem Heiligtum benutzt worden sein [37].

Im ptolemäischen Alexandria entstand auch ein Spiel, dessen Spielsteine Elisabeth Alfoeldi-Rosenbaum nach einer ersten Studie von Michael Rostovzeff aus verschiedenen Sammlungen und Einzelfunden zusammenstellte [38]. Auf seinen tesserae finden sich die Bezeichnungen und Darstellungen von Gottheiten ägyptisch-griechischer Herkunft, Personen und Lokalitäten. Warum das einzelne Motiv gewählt wurde,

[33] VAN MINNEN 1994.

[34] VON FALCK \& LICHTWARK 1996, p. 191 Nr. 184.

[35] Zu Puppen vergleiche JANSSEN 2003-4, FLUCK 2004 (dort p. 383-386 zu der genannten Puppe) und BEHLING 2011. Vgl. generell PARCA 2013, p. 473: „Toys as tools of cultural and gender identity constitute a vast and complex subject; however, the scarcity of materials of Ptolemaic date dissuades me from pursuing the topic here". Die bei Froschauer \& Harrauer 2004

genannten und gezeigten Objekte sind zumeist weder im Hinblick auf ihre Zeitstellung noch ihren Fundort noch ihre Zweckbestimmung präzis einzuordnen.

[36] PEDRIZET 1931; zuletzt zu dem Würfel PLATZ-HoRSTER 2017, p. 120, 163 f. Die Texte auch bei SEG 8, 811 und SB V 8234.

[37] Minas-NerPel 2007; vgl. jetzt auch Platz-HorsTER 2017, p. 118 , p. 144.

[38] AlföLdi-Rosenbaum 1976. 
lässt sich nicht immer sicher identifizieren. Insgesamt verweisen die Bezeichnungen aber klar auf das ptolemäische Ägypten und einzelne, wie z.B. Kanopos, ganz speziell auf Alexandria. Da einer Zahl das augusteische Legionslager Nikopolis zugeordnet wurde und einer anderen die Institution des "Kaisareions”, ist einerseits klar, dass das Spiel nach dem Sieg Oktavians über Mark Anton zumindest noch einmal verändert wurde. Andererseits fanden sich tesserae im Legionslager Vindonissa und in Pompeji, so dass es auch zweifelsfrei bereits in tiberischer Zeit (und beim Vesuvausbruch) in den verschiedenen Teilen des Reiches verbreitet war. Dass das Spiel ergänzt, aber nicht erst geschaffen wurde, liegt auch deshalb nahe, weil manchen tesserae nicht immer der gleiche Zahlenwert, aber immer ein verwandter zugewiesen wurde. Offensichtlich hat man in eine bestehende Abfolge einzelne neue Motive eingefügt. Die Verbreitung dieses Spieles - von Vindonissa über Bolsena, die Vesuvstädte, Athen, Kreta, Kleinasien (Tarsus) bis hin ans Schwarze Meer - ist ein besonders gutes Beispiel wie ein Spiel im gesamten römischen Reich verbreitet war und dabei in den verschiedensten Regionen und Milieus heimisch werden konnte.

\section{DIE LITERARISCHEN ZEUGNISSE}

Auch in den Werken in Ägypten tätiger Schriftsteller finden sich einzelne Spuren von Spielen [39]. Besonders wichtig sind sie natürlich, wenn sie solche erwähnen, die archäologisch nicht zu fassen sind. So wird z.B. den Juden in Alexandria in der Zeit Traians im Talmud vorgeworfen, dass sie so sehr die römische Vorliebe für Ballspiele übernommen hätten, dass sie selbst am Sabbat Ball gespielt hätten [40]. Häufiger kommt der alexandrinische Theologe Clemens an der Wende vom 2. zum 3. Jh. n. Chr. auf Spiele zu sprechen. Was er von Spielen erwähnt, dürfte zumindest zum Teil auf Beobachtungen vor Ort fußen, so seine Angaben über harpastum, ein rugbyartiges Ballspiel [41]. Dass seine Kritik am Würfeln mit Astragalen oder sechsseitigen Würfeln - für ihn und seinen Schüler Origenes waren Würfler die Betrüger schlechthin [42] - nicht rein theoretisch war, zeigen die vorgestellten Würfelfunde aus
Ägypten. Wie so oft, darf man eine solch theologische Ansicht aber nicht ungefragt als die Position aller Christen auffassen. Bischof Synesios von Kyrene, der als philosophisch geprägter Angehöriger der lokalen Führungsschicht zwar auch nicht unbedingt der typische Bischof war, schrieb in einem Brief (ep. 105) zu Beginn des 5. Jh. n. Chr.: "Ich teile meine Zeit in Spiel und geistige Arbeit ein. Beim theologischen Studium gehöre ich mir allein, beim Spiel allen. Du weißt nämlich, dass ich nach den Büchern zu jeder Art von Spielen neige". Mit inm fassen wir noch einmal einen Vertreter der sozialen Führungsschichten, deren Leben in allen seinen Aspekten und damit auch im Spielen einerseits generell quellenmäßig vergleichsweise gut bezeugt ist, bei denen es aber auch gute Gründe für die Annahme gibt, dass sie tatsächlich im überproportional großem Maße gespielt haben. Die andere Gruppe, für die das Gleiche gilt, die Soldaten, erscheinen in der nach 431 n. Chr. geschriebenen Paraphrase des Johannes-Evangelium des Nonnos von Panopolis. Dort findet sich zum erstenmal in einer literarischen Quelle die Vorstellung, die am Kreuz Jesu Wache haltenden Soldaten hätten um seine Kleidung gelost - eine Vorstellung, die in der gleichen Zeit auch auf Sarkophagen auftaucht [43]. Unsere Kenntnisse vom Spielen und den Spielen ist also auch im Falle des griechisch-römischen Ägyptens trotz einer so einzigartigen Quellengattung wie den Papyri und einer Reihe dank der besonderen klimatischen Bedingungen reichsweit singulärer archäologischer Funde recht fragmentarisch. Zweifellos ergibt sich aus beidem schon ein viel detaillierteres Bild, als es für die meisten Provinzen des Imperium Romanums der Fall ist. Aber von einem wirklich flächendeckenden und präzisen Einblick sind wir auch in dieser Provinz noch weit entfernt.

[39] Vgl. generell vor allem MENDNER 1978.

[40] Krauss 1912, p. 109 f. Zu Bällen aus Ägypten HÜBNER 1992, p. 40 f.

[41] Clem. Alex. paed. 3, 50, 1.

[42] Clem. Alex. paed. 3, 75, 2 bzw. Orig. comm. Ioh. 2, 15 (207,17 ed. Preuschen = 510, 27 ed. Blanc).

[43] Nonnos von Panopolis, Paraphrase des JohannesEvangelium in Hexametern 19, 24. Zu den archäologisch fassbaren Darstellungen: DE WAAL 1894. 
Alföldi-Rosenbaum, Elisabeth, 1976, „Alexandriaca. Studies on Roman Game Counters III”, Chiron 6, p. $205-239$. Bagnall, Roger \& Cribiore, Raffaella, 2006, Women's Letters from Ancient Egypt: 300 B.C.-A.D. 800, Ann Arbor. Bagnall, Roger S. \& RAthbone, Dominic W., 2004, Egypt. From Alexander to the Copts. An Archaeological and Historical Guide, London.

Behling, Claudia-Maria, 2011, „Puppen, Tiere und der Ernst des Lebens. Zum kulturhistorischen Aussagewert von Puppen und Tierfiguren aus spätantiker und frühchristlicher Zeit", Mitteilungen zur christlichen Archäologie 17, p. 91-104. BRUN, Jean-Pierre, 2003, "Dawwî", in: H. Cuvigny (ed.), La route de Myos Hormos. L'armée romaine dans le désert Oriental d'Egypte, Le Caire, I p. 133-136.

Carretero Vaquero, Santiago, 1998, "El ludus latrunculorum, un juego de estrategia practicado por los equites del ala II Flavia", Boletin del Seminario de Estudios de Arte y Arqueologia 64, p. 117-140.

Crist, Walter \& Dunn-Vaturi, Anne-Elizabeth \& De Voogt, Alex (Ed.), 2016, Ancient Egyptians at Play, London. Cuvigny, Hélène, 2011, Didymoi. Une garnison romaine dans le désert Oriental d'Egypte, I. Les fouilles et le matériel, Le Caire.

DeCKER, Karl Viktor, 1972, „Römisches Spielbrett und Spielgerät im Mittelrheinischen Landesmuseum Mainz", Bonner Hefte zur Vorgeschichte 3, p. 19-23.

Von FALCK, Martin \& LiChtwaRK, Friederike (Red.), 1996, Ägypten. Schätze aus dem Wüstensand. Kunst und Kultur der Christen am Nil. Katalog zur Ausstellung, Wiesbaden.

Ferrua, Antonio, 2001, Tavole lusorie epigrafiche, Roma.

Fields, Nic, 2003, Hadrian's Wall AD 122-410, Oxford.

FIтTÀ, Marco, 1998, Spiele und Spielzeug in der Antike. Unterhaltung und Vergnügen im Altertum, Stuttgart.

FLUCK, Cäcilia, 2004, ,"̈̈gyptische Puppen aus römischer und frühbyzantinischer Zeit. Ein Streifzug durch die Sammlungen des Ägyptischen Museums in Berlin, des Museums für angewandte Kunst in Wien und des Benaki-Museums in Athen", in: Hermann Harrauer \& Rosario Pintaudi (Hg.), Gedenkschrift Ulrike Horak (P. Horak), 2. Teil, Firenze, p. 383-400.

Froschauer, Harald \& Harrauer, Hermann, 2004, Spiel am Nil. Unterhaltung im Alten Ägypten, Wien.

Gates-Foster, Jennifer, 2012, "The Eastern Desert and the Red Sea Ports", in: Charlotte Riggs (ed.), The Oxford Handbook of Roman Egypt, Oxford, p. 736-748.

HAENSCH, Rudolf, 2012, "The Roman Army in Egypt", in: Charlotte Riggs (ed.), The Oxford Handbook of Roman Egypt, Oxford, p. 68-82.

HANeL, Norbert, 1997, "Sonderkeramik der Ziegelei? Eine tabula lusoria mit Mühlespiel und Legionsstempel”, Kölner Jahrbuch 30, p. $317-320$.

Holliger, Claudia \& Holliger, Christian, 1983, "Römische Spielsteine und Brettspiele", Jahresbericht der Gesellschaft Pro Vindonissa, p. 5-24.

HüBNER, Ulrich, 1992, Spiele und Spielzeug im antiken Palästina, Göttingen.

HÜBSCH, Gerbert, 1968, Die Personalangaben als Identifizierungsvermerke im Recht der gräko-ägyptischen Papyri, Berlin. JANSSEN, Rosalind M., 1996, "Soft toys from Egypt", in: Donald M. Bailey (ed.), Archaeological Reseach in Roman Egypt. The Proceedings of The Seventeenth Classical Colloquium of Greek and Roman Antiquities, British Museum, held on 1-4 December, 1993, Ann Arbor, p. 231-239.

Jördens, Andrea, 2001, „Papyri und private Archive. Ein Diskussionsbeitrag zur papyrologischen Terminologie”, in: Eva Cantarella \& Günther Thür (Hg.), Symposion 1997, Köln, Wien, Weimar, p. 253-268.

JöRDENS, Andrea, 2009, Statthalterliche Verwaltung in der römischen Kaiserzeit, Studien zum praefectus Aegypti, Stuttgart. KARUSU, Semni, 1973, "Der Erfinder des Würfels. Das älteste griechische mythische Porträt”, Athenische Mitteilungen 88, p. 55-65.

KIDD, Stephen E., 2017, "How to gamble in Greek: The meaning of kubeia”, Journal of Hellenic Studies 137, p. 119-134. Krauss, Samuel, 1912, Talmudische Archäologie III, Leipzig, p. 102-113.

LAMER, Hans, 1927, s. v. Iusoria tabula, RE XIII 2, col. 1900-2029.

MARCATTILI, Francesco, 2005, "Schola", in: Thesaurus cultus et rituum antiquorum, IV. Cult places. Representations of Cult places, Los Angeles, p. 319.

Matelly, Marie-Agnès, 2003, "Les petits objets", in: Hélène Cuvigny (ed.), La route de Myos Hormos. L'armée romaine dans le désert Oriental d'Egypte, Le Caire, II, p. 589-618.

Mendner, Siegfried, 1978, s. v. Gesellschaftsspiele, Reallexikon für Antike und Christentum X, col. 847-895.

MinAS-NerPEL, Martina, 2007, „A Demotic Inscribed Icosahedron from Dakleh Oasis”, Journal of Egyptian Archaeology 93, p. $137-148$.

VAN Minnen, Peter, 1994, "House-to-House Enquiries: An Interdisciplinary Approach to Roman Karanis", Zeitschrift für Papyrologie und Epigraphik 100, p. 227-251. 
Mulvin, Lynda \& Sidebotham, S. E., 2004, „Roman Game Boards from Abu Sha'ar (Red Sea Coast, Egypt)", Antiquity 78, p. 602-617.

OrRieuX, Claude, 1983a, Les papyrus de Zénon, L'horizon d'un Grec en Egypte au III. s. av. J.-C., Paris.

OrRIEUX, Claude, 1983b, Zénon de Caunos, parépidèmos, et le destin grec, Paris.

PARCA, Marlyne, 2013, "Children in Ptolemic Egypt: What the papyri say", in: Judith Evans Grubbs \& Tim Parkin (ed.), The Oxford Handbook of Childhood and Education in the Classical World, Oxford, p. 465-483.

Pedrizet, Paul, 1931, „Le jeu alexandrin de l'icosaèdre", Bulletin de I'Institut Francaise d'Archéologie Orientale 30, p. 1-16. Platz-Horster, Gertrud, 2017, „Antike Polyeder. Vom Spiel mit Form und Zahl im ptolemäischen Ägypten zum Kleinod im römischen Europa", Jahrbuch des Deutschen Archäologischen Instituts 132, p. 107-185.

ScнмID, Elisabeth, 1978, "Beinerne Spielwürfel von Vindonissa", Jahresbericht der Gesellschaft Pro Vindonissa, p. 54-81. Schmidt Heidenreich, Christophe, 2013, Le Glaive et l'Autel. Camps et piété militaires sous le Haut-Empire romain, Rennes.

Sperdel, Michael Alexander, 1996, Die römischen Schreibtafeln von Vindonissa. Lateinische Texte des militärischen Alltags und ihre geschichtliche Bedeutung, Baden-Dättwil.

STEINer, Paul, 1939, „Römisches Brettspiel und Spielgerät aus Trier”, Saalburg Jahrbuch 9, p. 34-45.

VoGT, Ulrich, 2012, Der Würfel ist gefallen. 5000 Jahre rund um den Kubus, Hildesheim u. a.

DE WAAL, Anton, 1894, Das Mora-Spiel auf Darstellungen der Verlosung des Kleides Christi, Römische Quartalsschrift 8 , p. $145-146$. 